\title{
REGION-BASED CLASSIFICATION OF REMOTE SENSING IMAGES WITH THE MORPHOLOGICAL TREE OF SHAPES
}

\author{
Gabriele Cavallaro $^{1}$, Mauro Dalla Mura ${ }^{2}$, Edwin Carlinet ${ }^{3,4}$ \\ Thierry Géraud ${ }^{3}$, Nicola Falco ${ }^{1}$, Jón Atli Benediktsson ${ }^{1}$ \\ ${ }^{1}$ Faculty of Electrical and Computer Engineering, University of Iceland, Reykjavik, Iceland \\ ${ }^{2}$ GIPSA-lab, Grenoble Institute of Technology, Grenoble, France \\ ${ }^{3}$ EPITA Research and Development Laboratory (LRDE), Le kremlin-Bicêtre, France \\ ${ }^{4}$ Université Paris-Est, LIGM, A3SI, ESIEE Paris, Noisy-le-Grand, France
}

\begin{abstract}
Satellite image classification is a key task used in remote sensing for the automatic interpretation of a large amount of information. Today there exist many types of classification algorithms using advanced image processing methods enhancing the classification accuracy rate. One of the best state-of-the-art methods which improves significantly the classification of complex scenes relies on Self-Dual Attribute Profiles (SDAPs). In this approach, the underlying representation of an image is the Tree of Shapes, which encodes the inclusion of connected components of the image. The SDAP computes for each pixel a vector of attributes providing a local multiscale representation of the information and hence leading to a fine description of the local structures of the image. Instead of performing a pixel-wise classification on features extracted from the Tree of Shapes, it is proposed to directly classify its nodes. Extending a specific interactive segmentation algorithm enables it to deal with the multi-class classification problem. The method does not involve any statistical learning and it is based entirely on morphological information related to the tree. Consequently, a very simple and effective region-based classifier relying on basic attributes is presented.
\end{abstract}

Index Terms - Remote Sensing, Classification, Mathematical Morphology, Tree of Shapes.

\section{INTRODUCTION}

The development of the latest-generation optical imaging sensors mounted on board of both terrestrial and satellite Earth observation platforms have led to the increasing availability of data with high spatial resolution. Very High Resolution (VHR) remotely sensed images provide a detailed representation of the surveyed scene with a geometrical resolution that at present can be up to $30 \mathrm{~cm}$ (WorldView-3). One of the most promising strategy for the analysis and the interpretation of the spatial information of a scene relies on hierarchical representations of the content of an image, which are available for example in the mathematical morphology framework.

The Tree of Shapes (ToS) [1,2] is a morphological tree that represents images in a self-dual and contrast invariant way. Due to the versatility of its structure, the ToS has proven to be a suitable representation for addressing many applications, such as visualization [3],

This research was supported in part by the program J. Verne 2015, project $n^{\circ} 31936 T D$ and by EU FP7 Theme Space project North State. The authors are grateful to F. Pacifici for providing the Rome data set. filtering [4],object detection [5], segmentation [6] and image simplification [7].

In the field of remote sensing, the ToS is mainly used for producing a multilevel filtering of images (e.g., from panchromatic, multispectral, hyperspectral). When these filtered images are considered as features for land cover classification, state-of-art results are obtained. The Self-Dual Attribute Profiles (SDAPs) [8], were proposed as a version of the Attribute Profiles (APs) [9] based on connected operators [10] computed on the ToS instead of considering a min and max-trees [10]. The use of the ToS allows simultaneously the processing of bright and dark regions of the image, leading consequently to a greater simplification of the image with respect to non self-dual filters. The basic idea of SDAPs is to study pixel-wise the behavior of a morphological operator at different levels of filtering. Specifically, a set of self-dual attribute filters are computed at different predefined thresholds that yields the feature space on which the classification is performed $[11,12]$. If, on the one hand, SDAPs provide meaningful features which allow classifiers to achieve remarkable classification results, on the other, their effectiveness is strictly dependent on the selected set of the filter parameters. Recently, the authors in [13] proposed a novel strategy for the automatic selection of the thresholds aiming at providing representative SDAPs. The resulted image decompositions were non-redundant and representative, and the salient structures were extracted. While the profiles are automatically generated, they are used for pixel-wise classification. The zones represented by the nodes of the ToS are not used. In this paper, the structure of the ToS is exploited and a classification based on regions is presented.

Nonetheless, the idea of this work started by a simple consideration: the filtered images which compose the SDAPs are computed by pruning the ToS. Thus, performing the classification directly on the $\operatorname{ToS}$ (i.e., classification of the nodes) should provide comparable results of the SDAPs since they carry information which is present in the ToS. The adopted scheme is the interactive segmentation algorithm proposed in [14] for the Multivariate Tree of Shapes [15, 16]. The algorithm is extended to a multi-class classification problem, with multispectral data sets acquired by QuickBird and IKONOS sensors over urban areas. The preliminary experiments are conducted by processing each image separately (i.e., channel-wise) with the ToS. It is shown that the method does not involve any statistical learning and it is based entirely on morphological information related to the tree such as gray levels or attribute values of the nodes. 


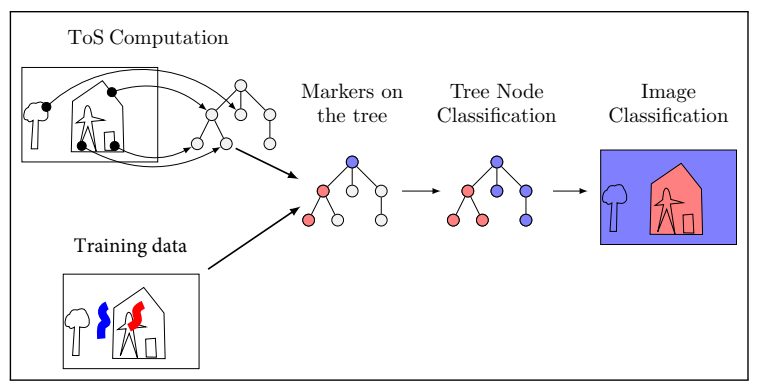

Fig. 1: Simplified scheme of the band-wise classifier.

\section{TREE OF SHAPES}

A self-dual tree has been defined in [2], called the Tree of Shapes (also known as inclusion tree), that describes the image contents in a unique way; the ToS can be interpreted as the result of merging the min- and max-tree [10] of the same image. The ToS is a morphological self-dual representation of the connected components within an image (i.e., zones enclosed by an isolevel line), also referred to as flat zones. It was firstly introduced by Monasse et al. [2], where the structure was computed with the Fast Level Line Transform (FLLT) algorithm: it first computes the pair of dual component trees and then obtains the ToS by merging both trees. Afterwards, Caselles et al. [17] introduced the Fast Level Set Transform algorithm (FLST), which relies on a region-growing approach to decompose the image into shapes. An operation called saturation is applied to the connected components which gives flat regions obtained by progressively merging nested regions. Specifically, the algorithm extracts each branch of the tree starting from the leaves and growing them up to the root until only a single flat region is reached. Song et al. [18], proposed to retrieve the ToS by building the tree of level lines and exploiting its interior of each level line. Recently Geraud et al. [19] proposed a new algorithm to compute the ToS in order to reduce the computational complexity and overcome the restriction to only $2 \mathrm{D}$ images of the previous methods. The algorithm computes the ToS with quasi-linear time complexity when data quantization is low (typically 12 bits or less) and it works for $\mathrm{nD}$ images. Moreover, Crozet et al. [20] presented the first parallel algorithm to compute the morphological ToS based on the previous algorithm [19].

Described more formally, let $f: \Omega \rightarrow E$ be a discrete twodimensional grayscale image, defined on a spatial domain $\Omega \subseteq \mathbb{Z}^{2}$ and taking values on a set of scalar values $E \subseteq \mathbb{Z}$ with an ordering relation $\leq$. For any $\lambda \in \mathbb{Z}$, a lower $\mathcal{L}(f)$ and upper $\mathcal{U}(f)$ threshold set is defined by:

$$
\begin{aligned}
& \mathcal{L}(f)=\{x \in \Omega, f(x)<\lambda\}, \\
& \mathcal{U}(f)=\{x \in \Omega, f(x)>\lambda\},
\end{aligned}
$$

Given $X \in P$ the power set of $E$, the set of connected components of $X$ is denoted as $\mathcal{C C}(X)$. If $\leq$ is a total relation, any two connencted components $X, Y \in \mathcal{C C}(\mathcal{L}(f))$ are either disjoint or nested. The min-tree and max-tree structures represent the components in $\mathcal{L}(f)$ and $\mathcal{U}(f)$ respectively with their inclusion relations. Given the hole-filling operator $\mathcal{H}$, a shape is any element of the set:

$$
\mathcal{S}=\{\mathcal{H}(\Gamma), \Gamma \in \mathcal{C C}(\mathcal{L}(f)) \cup \mathcal{C C}(\mathcal{U}(f))\}_{\lambda}
$$

If $\leq$ is total, any two shapes are either disjoint or nested, hence the cover of $\mathcal{S}, \subseteq$ form the Tree of Shapes.

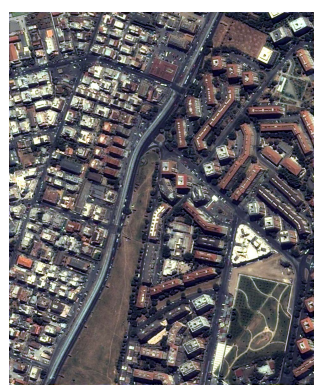

(a)

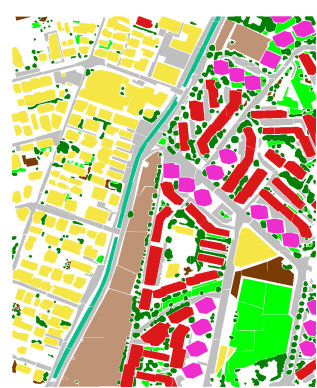

(b)

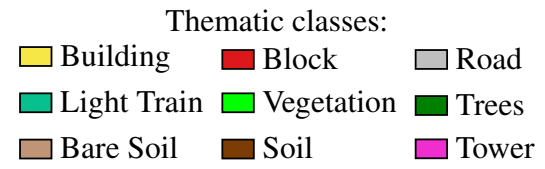

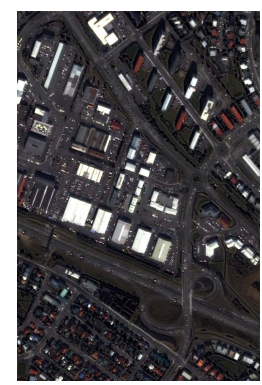

(c)

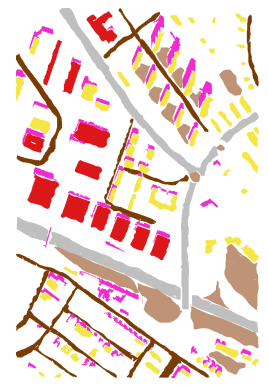

(d)
Thematic classes:

$\square$ Small building $\square$ Open area

$\begin{array}{ll}\square \text { Shadow } & \square \text { Large building } \\ \square \text { Large road } & \square \text { Street }\end{array}$

Fig. 2: QuickBird Rome (true color image (a) and groundtruth (b)) and IKONOS Reykjavik (true color image (c) and groundtruth (d)).

\section{SUPERVISED CLASSIFICATION OF THE TOS}

The multi-class classification problem is addressed with an extension of the interactive segmentation algorithm proposed in [14]. In this phase of preliminary experiments, the classification of the nodes is performed on the ToS (i.e., channel-wise) instead of using the Multivariate Tree of Shapes [15]. The classification is not pixel-based, since the classifier does not work directly on the image domain but in the space of the shapes (i.e., connected components), where the nodes are classified. The approach is based on Nearest Neighbor classification of nodes, in which neighboring nodes are defined according to the tree topology. In the following, the steps of the method are summarized (see also Fig. 1):

1. Compute the Tree of Shapes $T(f)$ of a gray-scale image $f$.

2. Estimate an attribute-based distance $\delta$ of each pair of connected nodes in the tree. The distance can be computed for example as a difference of gray-levels, areas, etc.

3. Mark the nodes according to the input training data. If a node gets labeled with different classes, the majority class is assigned,

4. Compute for each non-labeled node $S$ the $n$ distances 
Table 1: QuickBird Rome classification accuracies (mean value with its standard deviation in brackets) of each band for different distances

\begin{tabular}{ccccccc}
\hline Distance & Pan & Red & Green & Blue & Near Infrared & Majority vote \\
\hline \multirow{5}{*}{$\delta_{\text {val }}$} & $87.72(0.09)$ & $91.84(0.09)$ & $88.49(0.11)$ & $90.31(0.08)$ & $86.84(0.07)$ & $\mathbf{9 3 . 9 6 ( 0 . 1 1 )}$ \\
& $89.69(0.08)$ & $93.12(0.07)$ & $90.32(0.09)$ & $91.86(0.07)$ & $88.94(0.06)$ & $\mathbf{9 4 . 9 2 ( 0 . 0 8 )}$ \\
& $87.71(0.15)$ & $92.87(0.09)$ & $89.58(0.08)$ & $90.61(0.09)$ & $86.66(0.11)$ & $\mathbf{9 3 . 3 4}(\mathbf{0 . 0 9})$ \\
$\delta_{\text {att }(\text { area })}$ & $87.87(0.09)$ & $92.06(0.08)$ & $88.62(0.11)$ & $90.43(0.07)$ & $86.99(0.04)$ & $\mathbf{9 4 . 0 4 ( 0 . 1 1 )}$ \\
& $89.82(0.07)$ & $93.31(0.07)$ & $90.43(0.08)$ & $91.96(0.06)$ & $89.07(0.03)$ & $\mathbf{9 4 . 9 9 ( 0 . 0 8 )}$ \\
& $87.82(0.12)$ & $93.03(0.11)$ & $89.69(0.08)$ & $90.71(0.11)$ & $86.81(0.09)$ & $\mathbf{9 3 . 4 1 ( 0 . 1 2 )}$ \\
$\delta_{\text {att } \text { (inertia })}$ & $87.68(0.11)$ & $91.86(0.11)$ & $88.40(0.12)$ & $90.27(0.08)$ & $86.80(0.06)$ & $\mathbf{9 3 . 8 4 ( 0 . 1 1 )}$ \\
& $89.66(0.08)$ & $93.14(0.09)$ & $90.25(0.09)$ & $91.82(0.06)$ & $88.91(0.05)$ & $\mathbf{9 4 . 8 2 ( 0 . 0 8 )}$ \\
& $87.57(0.13)$ & $92.81(0.11)$ & $89.42(0.09)$ & $90.47(0.11)$ & $86.54(0.11)$ & $\mathbf{9 3 . 1 6 ( 0 . 0 9 )}$ \\
\hline
\end{tabular}

Table 2: IKONOS Reykjavik classification accuracies (mean value with its standard deviation in brackets) of each band for different distances

\begin{tabular}{ccccccc}
\hline Distance & Pan & Red & Green & Blue & Near Infrared & Majority vote \\
\hline \multirow{5}{*}{$\delta_{\text {val }}$} & $92.50(0.16)$ & $90.71(0.11)$ & $90.60(0.15)$ & $89.93(0.12)$ & $91.14(0.11)$ & $\mathbf{9 5 . 2 8}(\mathbf{0 . 0 8})$ \\
& $93.79(0.13)$ & $92.30(0.08)$ & $92.22(0.13)$ & $91.66(0.11)$ & $92.66(0.09)$ & $\mathbf{9 6 . 0 9}(\mathbf{0 . 0 6})$ \\
& $93.99(0.11)$ & $92.24(0.09)$ & $92.12(0.14)$ & $91.44(0.12)$ & $92.41(0.08)$ & $\mathbf{9 5 . 9 8}(\mathbf{0 . 0 6})$ \\
$\delta_{\text {att }(\text { area })}$ & $92.61(0.17)$ & $90.83(0.11)$ & $90.72(0.16)$ & $90.06(0.11)$ & $91.27(0.12)$ & $\mathbf{9 5 . 3 5}(\mathbf{0 . 0 7})$ \\
& $93.87(0.14)$ & $92.41(0.09)$ & $92.32(0.13)$ & $91.77(0.09)$ & $92.77(0.11)$ & $\mathbf{9 6 . 1 5}(\mathbf{0 . 0 6})$ \\
& $94.07(0.11)$ & $92.35(0.11)$ & $92.23(0.14)$ & $91.55(0.11)$ & $92.52(0.09)$ & $\mathbf{9 6 . 0 5}(\mathbf{0 . 0 6})$ \\
$\delta_{\text {att } \text { (inertia })}$ & $92.51(0.16)$ & $90.69(0.11)$ & $90.59(0.17)$ & $89.89(0.12)$ & $91.08(0.12)$ & $\mathbf{9 5 . 2 5}(\mathbf{0 . 0 9})$ \\
& $93.78(0.13)$ & $92.29(0.09)$ & $92.21(0.14)$ & $91.63(0.11)$ & $92.61(0.11)$ & $\mathbf{9 6 . 0 7}(\mathbf{0 . 0 8})$ \\
& $93.98(0.12)$ & $92.23(0.11)$ & $92.11(0.15)$ & $91.41(0.12)$ & $92.36(0.09)$ & $\mathbf{9 5 . 9 6 ( 0 . 0 8 )}$ \\
\hline
\end{tabular}

$\left\{d_{1}, d_{2}, d_{3}, \ldots, d_{n}\right\}$ to the closest corresponding $n$ classes.

(i) Initialize each $d_{i}$, with $i \in\{1,2,3, \ldots, n\}$, as 0 if $i$ is equal to the label of the node $S$, otherwise $+\infty$.

(ii) For each class i, from the leaves to the root, compute: $d_{i}(\operatorname{parent}(S))=\min \left(d_{i}(\operatorname{parent}(S)), d_{i}(S)+\right.$ $\delta(S, \operatorname{parent}(S)))$

(iii) For each class i, from the root to the leaves, compute: $d_{i}(S)=\min \left(d_{i}(S), d_{i}(\operatorname{parent}(S))+\delta(S\right.$, parent $\left.(S))\right)$

5. Classify every non-labeled node with the closest marked node $\min _{c \in\{1,2,3, \ldots, n\}} d_{c}(S)$.

6. Compute the classification map by retrieving the image from the labels of $T(f)$ (i.e., each pixel which belongs to a single node is set to the label assigned to the node).

\section{EXPERIMENTAL RESULTS}

The first dataset used in our experiments is an image of Rome, Italy, acquired by the QuickBird satellite. The dataset is composed of a low-resolution $(2.4 \mathrm{~m})$ multispectral image with four bands Red, Green, Blue and Near Infrared and a high spatial resolution panchromatic image of $0.6 \mathrm{~m}$ resolution. This dataset is considered challenging due to the oblique acquisition angle and the presence of long shadows. The second dataset is an image of Reykjavik, Iceland, acquired by the IKONOS Earth imaging satellite. As with the other dataset, it consists of a low-resolution (4m) multispectral image with the four bands and a high spatial resolution panchromatic image of $1 \mathrm{~m}$ resolution. For each data set the panchromatic and multispectral images are pansharpened using the undecimated discrete wavelet transform (UDWT) method [21], and the obtained high-resolution multispectral images are used for the classification. The true-colour images and the groundtruth data are shown in Fig. 2.

Given a dataset, the classification is performed on each single image (i.e., spectral band), as described in Section 3. Each experiment is repeated ten times, randomly selecting $10 \%$ of the references samples as training set, and the mean values of the overall (OA), average (AA) accuracy and kappa (K) coefficient are given. In the experiments, three different measures $\delta$ (see Section 3 ) were considered as distances between two nodes : $\delta_{\text {val }}$ difference of their gray-level, $\delta_{a t t(\text { area })}$ difference of their area attribute values, and $\delta_{\text {att(inertia) }}$ difference of their moment of inertia attribute values.

The classification process does not involve any statistical learning, and the attribute-based distances computed on the ToS are the only information available to the classifier for making a decision. The preliminary classification results reported in Table 1 and Table 2, for the Rome and Reykjavik data set, respectively, are promising. Despite the simplicity of the classifier considered in this work (i.e., nearest neighbor) and the use of a single feature (i.e., one spectral band), very good results are obtained for both datasets achieving an average OA of $93 \%$. The classification results can be improved with the fusion of the single results obtained for each spectral band using a majority voting strategy. This approach outperformed in terms of average OA the classification results obtained considering a single feature $(+1.96 \%$ and $+1.98 \%$ for the two datasets, respectively). Obviously, other fusion criteria can be applied.

In comparison with the results provided by the state-of-the-art methods ((APs) [9] and (SDAPs) [8][12]), the proposed method achieves comparable performance, yet slightly lower, without using sophisticated classification methods (such as SVM and Random Forest [9]) and several features. 


\section{CONCLUSION AND PERSPECTIVES}

A simple and effective region-based classification of remote sensing images method developed on the Tree of Shapes representation has been presented. Despite the simplicity of the Tree of Shapes structure, its versatility and potential allow for comparable performances as the currently used sophisticated classification algorithms. Instead of performing a pixel-based classification, the proposed method relies first on classifying the nodes of the tree. Then, each pixel, which belongs to a single node, is set to the label assigned to the node.

Additional experiments are needed to establish the performances of this approach, and for instance clarify why the morphological distance based on attribute area performs slightly better than the others. Fusion strategies between the distances may be required in order to improve the classification results. Moreover, statistical learning strategies may be integrated within the algorithm in order to improve the robustness of the classifier.

\section{REFERENCES}

[1] Vicent Caselles, Bartomeu Coll, and Jean Michel Morel, "Topographic Maps and Local Contrast Changes in Natural Images," International Journal of Computer Vision, vol. 33, no. 1, pp. 5-27, 1999.

[2] Pascal Monasse and Frederic Guichard, "Fast Computation of a Contrast-Invariant Image Representation," IEEE Transactions on Image Processing, vol. 9, no. 5, pp. 860-872, 2000.

[3] Yongchao $\mathrm{Xu}$, Edwin Carlinet, Thierry Géraud, and Laurent Najman, "Meaningful Disjoint Level Lines Selection," in Proceedings of the 21st International Conference on Image Processing (ICIP), Paris, France, 2014, pp. 2938-2942.

[4] Vicent Caselles and Pascal Monasse, "Grain Filters," Journal of Mathematical Imaging and Vision, vol. 17, no. 3, pp. 249270, 2002.

[5] Yongchao $\mathrm{Xu}$, Thierry Géraud, and Laurent Najman, "Context-Based Energy Estimator: Application to Object Segmentation on the Tree of Shapes," in Proceedings of the 19th International Conference on Image Processing (ICIP), Orlando, Florida, USA, 2012, pp. 1577-1580.

[6] Françoise Dibos and Georges Koepfler, "Total Variation Minimization by the Fast Level Sets Transform,” p. 179, jul 2001.

[7] Coloma Ballester, Vicent Caselles, Laura Igual, and Luis Garrido, "Level Lines Selection with Variational Models for Segmentation and Encoding," Journal of Mathematical Imaging and Vision, vol. 27, pp. 5-27, 2007.

[8] Mauro Dalla Mura, Jón Atli Benediktsson, and Lorenzo Bruzzone, "Self-dual Attribute Profiles for the Analysis of Remote Sensing Images," in Mathematical Morphology and Its Applications to Image and Signal Processing - Proceedings of the 10th Intl. Symp. on Mathematical Morphology (ISMM), vol. 6671 of Lecture Notes in Computer Science, pp. 320-330. Springer, 2011.

[9] Mauro Dalla Mura, Jón Atli Benediktsson, Bjórn Waske, and Lorenzo Bruzzone, "Morphological Attribute Profiles for the Analysis of Very High Resolution Images," IEEE Transactions on Geoscience and Remote Sensing, vol. 48, no. 10, pp. 37473762, 2010.

[10] Philippe Salembier, Albert Oliveras, and Luis Garrido, "Antiextensive Connected Operators for Image and Sequence
Processing," IEEE Transactions on Image Processing, vol. 7, no. 4, pp. 555-570, 1998.

[11] Gabriele Cavallaro, Mauro Dalla Mura, Jón Atli Benediktsson, and Lorenzo Bruzzone, "A Comparison of Self-Dual Attribute Profiles Based on Different Filter Rules for Classification," in Proceedings of the IEEE International Geoscience and Remote Sensing Symposium (IGARSS), Québec, Canada, 2014, pp. 1265-1268.

[12] Gabriele Cavallaro, Mauro Dalla Mura, Jón Atli Benediktsson, and Lorenzo Bruzzone, "Extended Self-Dual Attribute Profiles for the Classification of Hyperspectral Images," IEEE Geoscience and Remote Sensing Letters, vol. 12, no. 8, pp. 16901694, 2015.

[13] Gabriele Cavallaro, Nicola Falco, Mauro Dalla Mura, Lorenzo Bruzzone, and Jón Atli Benediktsson, "Automatic Threshold Selection for Profiles of Attribute Filters Based on Granulometric Characteristic Functions," in Mathematical Morphology and Its Application to Signal and Image Processing - Proc. of the 12th Intl. Symp. on Mathematical Morphology (ISMM), 2015, vol. 9082 of Lecture Notes in Computer Science, pp. 169-181.

[14] Edwin Carlinet and Thierry Géraud, "Morphological Object Picking Based on the Color Tree of Shapes," in Proceedings of the 5th International Conference on Image Processing Theory, Tools and Applications (IPTA'15), Orléans, France, 2015.

[15] Edwin Carlinet and Thierry Géraud, "A Color Tree of Shapes with Illustrations on Filtering, Simplification, and Segmentation," in Mathematical Morphology and Its Application to Signal and Image Processing - Proc. of the 12th Intl. Symp. on Mathematical Morphology (ISMM). 2015, vol. 9082 of Lecture Notes in Computer Science, pp. 363-374, Springer.

[16] Edwin Carlinet and Thierry Géraud, "MToS: A Tree of Shapes for Multivariate Images," IEEE Transactions on Image Processing, vol. 24, no. 12, pp. 5330-5342, 2015.

[17] Vicent Caselles and Pascal Monasse, Geometric Description of Images as Topographic Maps, vol. 1984 of Lecture Notes in Mathematics, Springer, 1 edition, 2009.

[18] Yuqing Song, "A Topdown Algorithm for Computation of Level Line Trees," IEEE Transactions on Image Processing, vol. 16, no. 8, pp. 2107-2116, 2007.

[19] Thierry Géraud, Edwin Carlinet, Sébastien Crozet, and Laurent Najman, "A Quasi-Linear Algorithm to Compute the Tree of Shapes of $n \mathrm{D}$ Images," in Mathematical Morphology and Its Application to Signal and Image Processing - Proc. of the 11th Intl. Symp. on Mathematical Morphology (ISMM). 2013, vol. 7883 of Lecture Notes in Computer Science, pp. 98-110, Springer.

[20] Sébastien Crozet and Thierry Géraud, “A First Parallel Algorithm to Compute the Morphological Tree of Shapes of $n \mathrm{D}$ Images," in Proceedings of the IEEE International Conference on Image Processing (ICIP), Paris, France, 2014, pp. 2933-2937.

[21] G.P. Nason and B.W. Silverman, "The Stationary Wavelet Transform and Some Statistical Applications," Wavelets and statistics, pp. 281-299, 1995. 